\title{
Histopathological examination of spine tumors after treatment with radiosurgery
}

\author{
Nathan T. Zwagerman, MD, ${ }^{1}$ Michael M. McDowell, MD, ${ }^{1}$ Ronald L. Hamilton, MD, ${ }^{2}$ \\ Edward A. Monaco III, MD, PhD, ${ }^{1}$ John C. Flickinger, MD, ${ }^{3}$ and Peter C. Gerszten, MD, MPH ${ }^{1}$ \\ Departments of ${ }^{1}$ Neurological Surgery and ${ }^{3}$ Radiation Oncology, and ${ }^{2}$ Division of Neuropathology, University of Pittsburgh, \\ Pennsylvania
}

OBJECTIVE Increased survival time after diagnosis of neoplastic disease has resulted in a gradual increase in spine tumor incidence. Radiosurgery is frequently a viable alternative to operative management in a population with severe medical comorbidities. The authors sought to assess the histopathological consequences of radiosurgery in the subset of patients progressing to operative intervention.

METHODS Eighteen patients who underwent radiosurgery for spine tumors between 2008 and 2014 subsequently progressed to surgical treatment. A histopathological examination of these cases was performed. Indications for surgery included symptomatic compression fractures, radiographic instability, and symptoms of cord or cauda equina compression. Biopsy samples were obtained from the tumor within the radiosurgical zone in all cases and were permanently fixated. Viable tumor samples were stained for Ki 67.

RESULTS Fifteen patients had metastatic lesions and 3 patients had neurofibromas. The mean patient age was 57 years. The operative indication was symptomatic compression in 10 cases (67\%). The most frequent metastatic lesions were breast cancer (4 cases), renal cell carcinoma (3), prostate cancer (2), and endometrial cancer (2). In 9 (60\%) of the 15 metastatic cases, histological examination of the lesions showed minimal evidence of inflammation. Viable tumor at the margins of the radiosurgery was seen in $9(60 \%)$ of the metastatic cases. Necrosis in the tumor bed was frequent, as was fibrotic bone marrow. Vascular ectasia was seen in 2 of 15 metastatic cases, but sclerosis with ectasia was frequent. No evidence of malignant conversion was seen in the periphery of the lesions in the 3 neurofibroma cases. In 1 case of neurofibroma, the lesion demonstrated some small areas of remnant tumor in the radiosurgical target zone.

CONCLUSIONS This case series demonstrates important histopathological characteristics of spinal lesions treated by SRS. Regions with the highest exposure to radiation appear to be densely necrotic and show little evidence of tumor growth, whereas peripheral regions distant from the radiation dosage are more likely to demonstrate viable tumor in malignant and benign neoplasms. Physiological tissue appears to be similarly affected. With additional investigation, a more homogenized field of hypofractionated radiation exposure may allow for tumor obliteration with relative preservation of critical anatomical structures.

http://thejns.org/doi/abs/10.3171/2016.5.FOCUS16119

KEY WORDS oncology; spine; radiosurgery; Gamma Knife; histopathology

A s our therapeutic acumen in the treatment of neoplastic diseases increases, there has been an inadvertent effect resulting in a growing number of patients living in the context of both primary and metastatic disease. A large fraction of patients with a malignant neoplasm may face metastasis to the spine alone, manifesting in over 150,000 new diagnoses of spinal metastases and primary spine tumors annually. 2,3,7,11,12 Aggressive interventions in patients with active oncological processes are often associated with high risk, and for this reason stereotactic radiosurgery (SRS) has been increasingly used for the treatment of both metastatic and primary spinal tumors. Although SRS results in many good clinical outcomes, inevitably there are cases of tumor progression and worsening symptoms. This study was performed to analyze the histopathological findings in a series of spine tumors treated with SRS that subsequently required surgical management, which provided tissue for analysis.

\section{Methods}

Between 2008 and 2014, 129 patients underwent SRS 
for the treatment of spinal neoplasms at our institution. Of these 129 patients, 18 subsequently received surgical treatment related to these lesions at the same institution. In all 18 cases, the surgery was performed by the senior author. All 129 patients received SRS via an Elekta Synergy S 6-MV linear accelerator with a beam modulator and cone beam CT imaging guidance combined with a HexaPOD couch. The patients who required open surgical treatment after SRS had developed compression fractures, instability, or symptoms of cord or cauda equina compression such as radiculopathy or myelopathy. Pathological samples were sent routinely in each case for permanent fixation in $10 \%$ neutral buffered formaldehyde and paraffin and were stained in hematoxylin and eosin (H \& E) before examination. Visibly viable tumor samples were stained for Ki 67 to assess for proliferative activity. All sampling occurred in regions previously exposed to SRS.

\section{Results}

The mean patient age of our 18-patient cohort was 57 years. Fifteen patients treated had diagnoses of metastatic disease, and 3 patients were treated for benign neurofibromas. Among the patients with metastatic disease, the operative indication was spinal cord or cauda equina compression in 10 (67\%). The remaining 5 patients (34\%) required surgical management via open instrumentation or percutaneous cement augmentation due to symptomatic fractures or instability. Magnetic resonance imaging findings consistent with tumor progression were seen in $10 \mathrm{pa}-$ tients (67\%). The most common histological tumor types represented by the metastatic lesions were breast cancer (4), renal cell carcinoma (3), prostate cancer (2), and endometrial cancer (2).

The metastatic lesions had a mean treatment volume of $42.6 \mathrm{~cm}^{3}$ with a mean maximum dose of $18 \mathrm{~Gy}$; the mean maximum point dose delivered was $20 \mathrm{~Gy}$. The patients with neurofibromas received a mean maximum dose of 18.6 Gy with a mean treatment volume of $6.2 \mathrm{~cm}^{3}$.

\section{Histopathological Findings}

On review of the histopathology of the 15 cases of spinal metastases, 9 cases $(60 \%)$ had minimal evidence of inflammation, 3 cases (20\%) had mild inflammation, and 1 case $(7 \%)$ had moderate inflammation. Frank regions of proliferative malignancy were uncommon, but some viable tumor was seen in 9 cases at the margins of radiotherapy $(60 \%)$ (Fig. 1) compared with the more frequent finding of radiation-induced soft tissue necrosis, osteonecrosis, sclerosis, and inflammation with interspersed regions of atypical cell clusters (Fig. 2). Necrosis within the tumor bed was seen in 9 cases $(60 \%)$. Necrosis and fibrosis of the soft tissues tended to be extensive and included paravertebral, muscular, and adipose tissues sampled (Fig. 3). Fibrotic bone marrow was seen in 9 cases (60\%). Regions of typical cells were subjected to immunohistochemical staining for high-and low-molecular weight cytokeratins, with positive results being consistent with abnormal carcinomatous replication in regions that typically are replication dormant (Fig. 4). ${ }^{5}$ Isolated vascular ectasia alone was rarely seen (evident in 2 of 15 cases), but sclerosis and

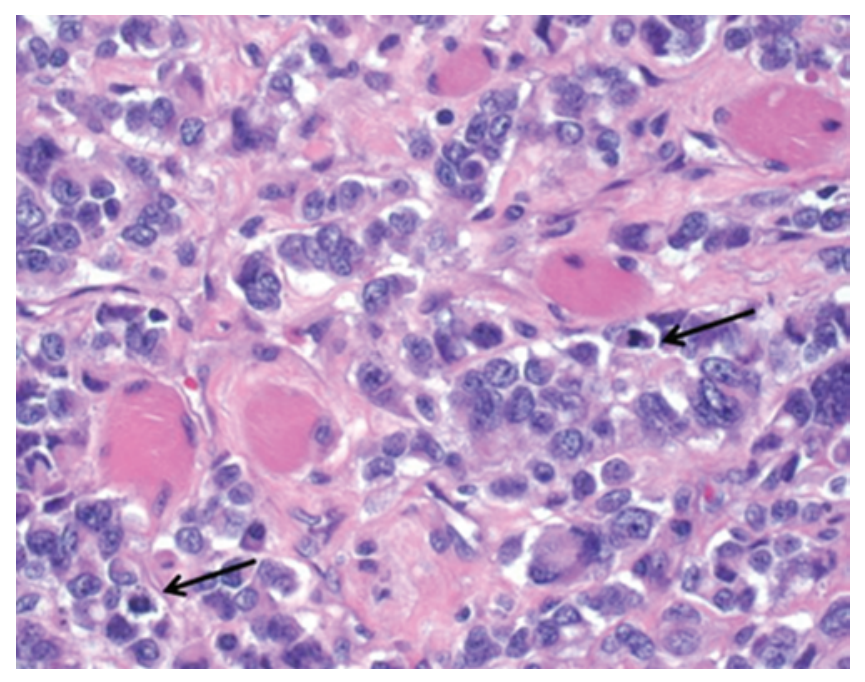

FIG. 1. Photomicrograph of a section of a recurrent metastatic breast carcinoma showing mitotic figures (arrows) identified at the margin of radiation therapy. $\mathrm{H} \& \mathrm{E}$, original magnification $\times 400$.

ectasia were seen frequently in tandem (Fig. 5). This is in contrast to the brain, where the finding of ectatic vessels after radiation is well documented. ${ }^{6,9,10}$

On review of the histopathology of the 3 cases of neurofibromas, no case had signs of malignant conversion. The majority of the histological slides demonstrated large central areas of sclerosis with bands of acellular material. One $(33 \%)$ of the 3 cases had sparsely cellular regions within the central necrosis and remnant myelinated axons (Figs. 6 and 7). In all 3 cases, the regions at the periphery of the SRS target zone demonstrated normal characteristics of neurofibromas. Overall, the histological characteristics were consistent with standard neurofibroma findings except in the 1 case represented in Figs. 6 and 7.

\section{Discussion}

The primary concern for patients presenting with new neurological symptoms in the context of a known spinal lesion treated by SRS is tumor progression despite radiotherapy. In our study, we found that evidence of large-scale active tumor was far less frequent than postradiation in-

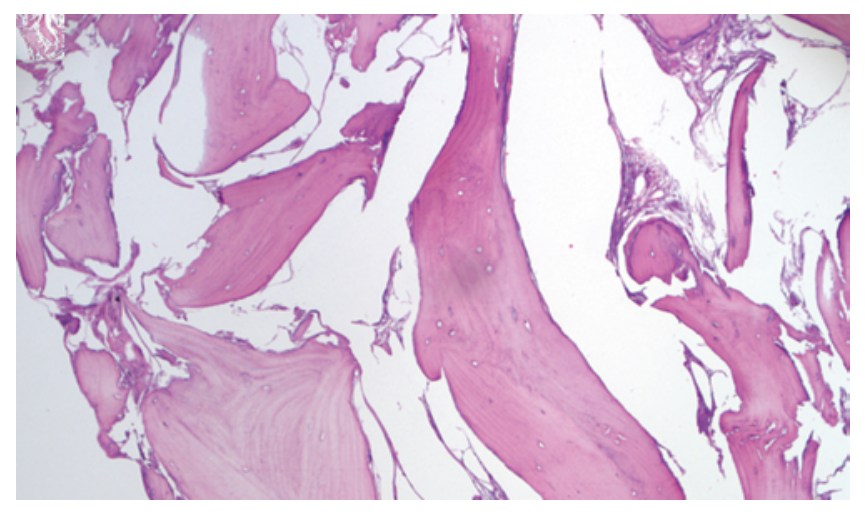

FIG. 2. Photomicrograph demonstrating osteonecrosis with complete loss of osteocyte nuclear staining and fibrosis of the marrow. $\mathrm{H} \& \mathrm{E}$, original magnification $\times 100$. 


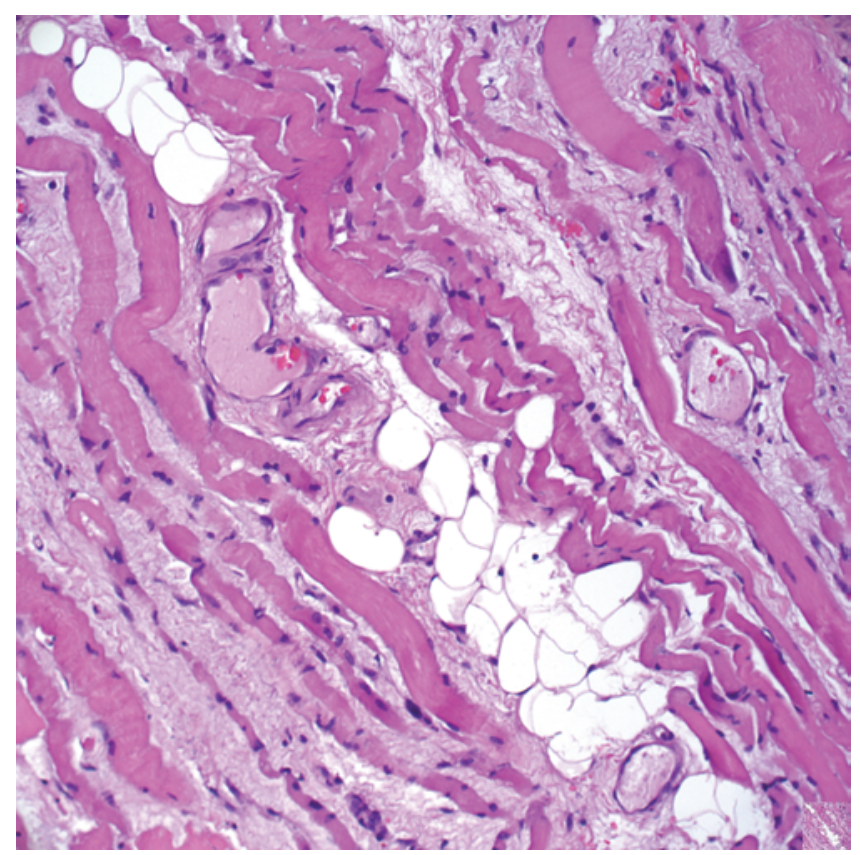

FIG. 3. Photomicrograph demonstrating radiation necrosis of skeletal muscle and adipose tissue with intervening interstitial fibrosis. $\mathrm{H} \& \mathrm{E}$, original magnification $\times 200$.

jury to abnormal and normal tissue alike. Inflammation was seen in all but 2 metastatic lesions, and injury to bony structures was prevalent. Spinal fracture secondary to SRS occurs in up to $40 \%$ of cases based on dosing. ${ }^{1,4,8}$ While 9 cases did show at least some active tumor, the regions of highest density were near the margins of the target region of radiation, suggesting that higher radiation doses near the periphery would have been equally tumoricidal, which

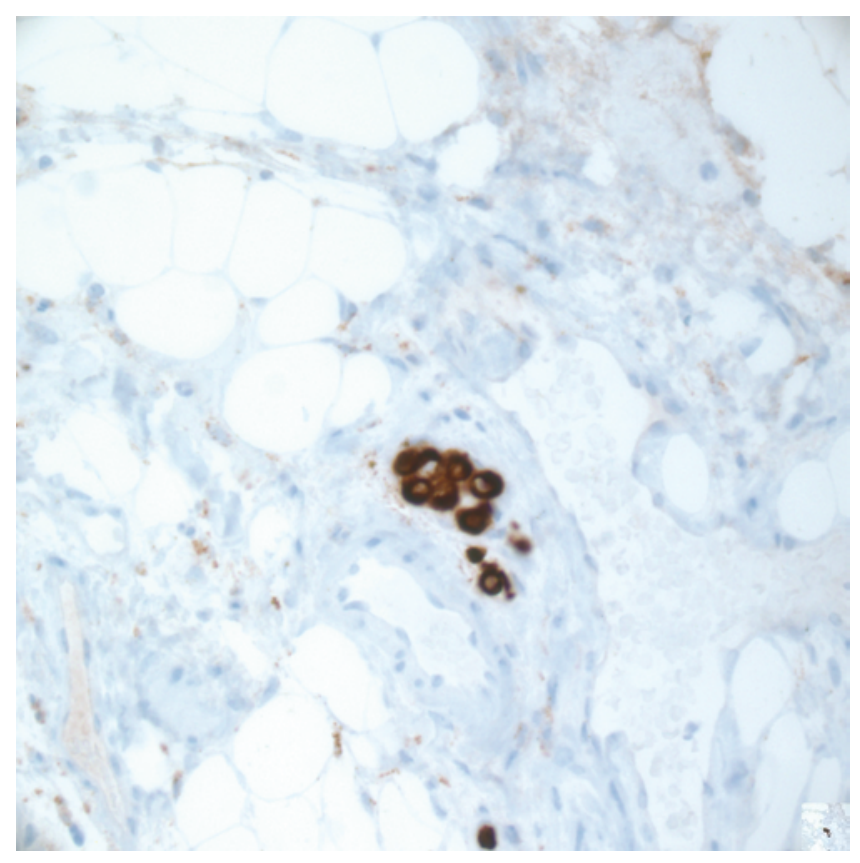

FIG. 4. Photomicrograph obtained after immunohistochemical staining for cytokeratin demonstrating a small cluster of residual carcinoma cells. Original magnification $\times 400$.

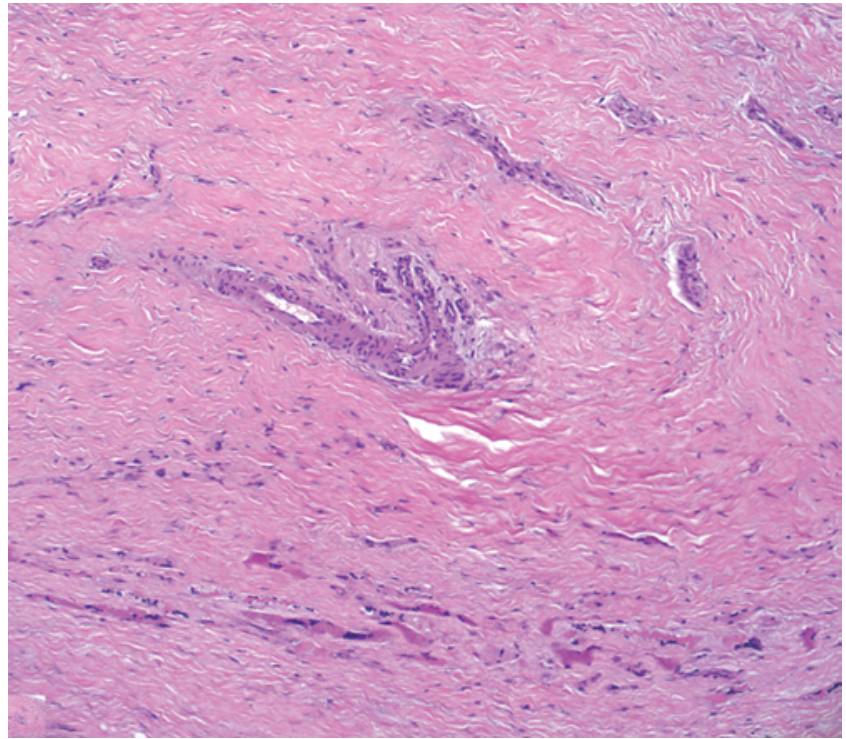

FIG. 5. Photomicrograph showing sclerosis of compacted vasculature with reduction in lumen volume. $\mathrm{H} \& \mathrm{E}$, original magnification $\times 40$.

is argued by the dense necrosis seen in the tumor bed of samples. Ectasia was seen in 2 metastatic cases and was felt to be as a result of radiation effect on intrinsic vasculature based on anatomical considerations.

The lack of more prevalent neovasculature may be due to the overwhelming intracellular injury within the nidus of radiation, as evidenced by the dense regions of necrosis seen. We speculate that the vascular changes demonstrated may be more reparative than vasculopathic. The paucity of malignant cells may also not be sufficient to generate angiogenesis on an appreciable level. Fibrosis within the bone marrow was frequently seen. High doses of radiation have been presumed to be causative with such findings, but the lack of changes in some specimens suggests that hypofractionated radiation therapy may allow for some preservation of marrow within the radiation zone.

The 3 cases of neurofibromas were notable for the absence of evidence of malignant conversion after SRS. This provides support for both the effectiveness of the appropri-

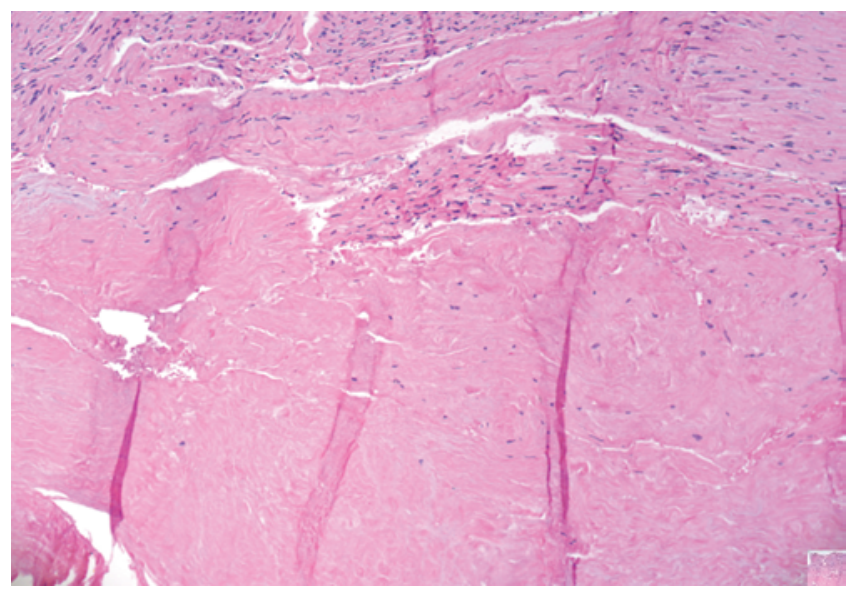

FIG. 6. Photomicrograph from neurofibroma showing central sclerosis and peripheral benign spindle cells with wavy nuclei amid degenerative inflammation. $\mathrm{H} \& \mathrm{E}$, original magnification $\times 40$. 


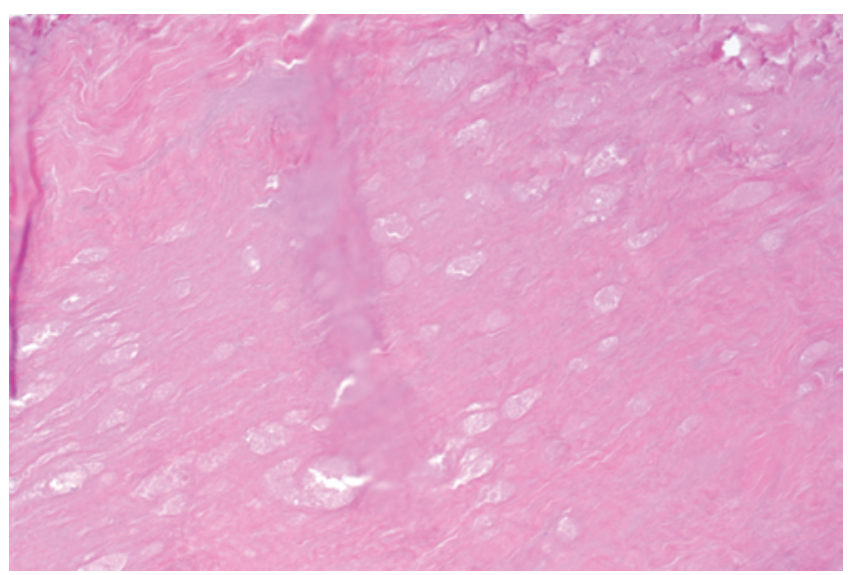

FIG. 7. Photomicrograph showing degenerating myelinated neuronal axons from necrotic neurofibroma cells. $\mathrm{H} \& \mathrm{E}$, original magnification $\times 40$.

ate dosage in the treatment of neurofibroma and suggests a relatively low risk of stimulating or permitting malignant conversion.

\section{Conclusions}

This case series demonstrates important histopathological characteristics of spinal lesions treated by SRS. Regions with the highest exposure to radiation appear to be densely necrotic and show little evidence of tumor growth, whereas peripheral regions distant from the radiation dosage are more likely to demonstrate viable tumor in malignant and benign neoplasms. Physiological tissue appears to be similarly affected. With additional investigation, a more homogenized field of hypofractionated radiation exposure may allow for tumor obliteration with relative preservation of critical anatomical structures.

\section{References}

1. Al-Omair A, Smith R, Kiehl TR, Lao L, Yu E, Massicotte EM, et al: Radiation-induced vertebral compression fracture following spine stereotactic radiosurgery: clinicopathological correlation. J Neurosurg Spine 18:430-435, 2013

2. Barron KD, Hirano A, Araki S, Terry RD: Experiences with metastatic neoplasms involving the spinal cord. Neurology 9:91-106, 1959

3. Black P: Spinal metastasis: current status and recommended guidelines for management. Neurosurgery 5:726-746, 1979

4. Boehling NS, Grosshans DR, Allen PK, McAleer MF, Burton AW, Azeem S, et al: Vertebral compression fracture risk after stereotactic body radiotherapy for spinal metastases. J Neurosurg Spine 16:379-386, 2012

5. Dabbs D: Diagnostic Immunohistochemistry, ed 1. Philadelphia: Churchill Livingstone, 2002

6. Gaensler EH, Dillon WP, Edwards MS, Larson DA, Rosenau W, Wilson CB: Radiation-induced telangiectasia in the brain simulates cryptic vascular malformations at MR imaging. Radiology 193:629-636, 1994

7. Gokaslan ZL, York JE, Walsh GL, McCutcheon IE, Lang FF, Putnam JB Jr, et al: Transthoracic vertebrectomy for metastatic spinal tumors. J Neurosurg 89:599-609, 1998

8. Rose PS, Laufer I, Boland PJ, Hanover A, Bilsky MH, Yamada J, et al: Risk of fracture after single fraction image-guided intensity-modulated radiation therapy to spinal metastases. $\mathbf{J}$ Clin Oncol 27:5075-5079, 2009

9. Rubin P, Gash DM, Hansen JT, Nelson DF, Williams JP: Disruption of the blood-brain barrier as the primary effect of CNS irradiation. Radiother Oncol 31:51-60, 1994

10. Valk PE, Dillon WP: Radiation injury of the brain. AJNR Am J Neuroradiol 12:45-62, 1991

11. Walsh GL, Gokaslan ZL, McCutcheon IE, Mineo MT, Yasko AW, Swisher SG, et al: Anterior approaches to the thoracic spine in patients with cancer: indications and results. Ann Thorac Surg 64:1611-1618, 1997

12. Yamada Y, Lovelock DM, Bilsky MH: A review of imageguided intensity-modulated radiotherapy for spinal tumors. Neurosurgery 61:226-235, 2007

\section{Disclosures}

The authors report no conflict of interest concerning the materials or methods used in this study or the findings specified in this paper.

\section{Author Contributions}

Conception and design: Gerszten, Zwagerman. Acquisition of data: Gerszten, Zwagerman, McDowell, Hamilton, Flickinger. Analysis and interpretation of data: Gerszten, Zwagerman, McDowell, Hamilton, Flickinger. Drafting the article: Zwagerman, McDowell, Monaco. Critically revising the article: all authors. Reviewed submitted version of manuscript: all authors. Approved the final version of the manuscript on behalf of all authors: Gerszten. Statistical analysis: Zwagerman. Administrative/technical/material support: Gerszten. Study supervision: Gerszten.

\section{Correspondence}

Peter C. Gerszten, Department of Neurological Surgery, University of Pittsburgh, 200 Lothrop St., Ste. B-400 PUH, Pittsburgh, PA 15213-2582. email: gersztenpc@upmc.edu. 